\title{
Prevalence and risk factors for Pulmonary Embolism (PE) and Deep Vein Thrombosis (DVT) during Acute Exacerbation of Chronic Obstructive Pulmonary Disease (AECOPD)
}

\author{
Corresponding author: \\ Daniela Buklioska llievska \\ "Nikola Parapunov" 31/1-3 Skopje, \\ Macedonia \\ e-mail: dbuklioska@yahoo.com
}

\begin{abstract}
Introduction: COPD patients are at high risk for PE and DVT due to immobility, inflammation, comorbidities Prevalence of PE during AECOPD is uncertain and often under-diagnosed.

Material and methods: Single-center, prospective, an observational trial of 100 hospitalized patients with AECOPD, diagnosed according to GOLD criteria, 40-75 years, stratified according to airflow limitation (I-IV), divided into subgroups (PE-diagnosed/non-PE and with known/ undetermined exacerbation etiology). Investigations: clinical risk assessment, electrocardiogram (ECG), laboratory, spirometry, gas-analysis, D-dimer (DD), chest X-ray, thoracic ultrasonography (TUS), Doppler-ultrasonography of deep-veins of lower-extremities (DULE). Patients with high DD and DVT or high DD and abnormal TUS underwent computed-tomography pulmonary-angiography (CTPA).

Results: PE was diagnosed in 26 (26.0\%), DVT in 5 (5.0\%) of hospitalized AECOPD patients. There was a positive correlation between COPD-severity and PE. Frequencies of PE in GOLD-stages I, IV, were 0 (0.0\%), 3 (11.5\%), 8 (30.7\%), 15 (57.7\%) respectively. Patients with pleuritic chest-pain, TUS abnormality, phlebitis and high DD were more likely to develop PE. Localization was subsegmental in 9 (34.6\%), in one of the main pulmonary arteries 7 (26.9\%), lobar and interlobar arteries in 10 (38.5\%). DD was significantly higher among patients with $P E$ than those without $(3.34 \pm 1.1 \mu \mathrm{g} / \mathrm{mL}$ vs. $2.2 \pm 0.8 \mu \mathrm{g} / \mathrm{mL}, \mathrm{P}<0.0001)$. There was positive correlation between the presence of $P E$ and elevated $D D>2.0 \mu \mathrm{g} / \mathrm{mL}(P=0.02)$. There was no statistically significant difference between patients with $\mathrm{PE}$ and without, according to age, gender and comorbidities $(P>0.05)$. Immobility and obesity were significantly higher among PE patients, $\mathrm{P}=0.032$ and $\mathrm{P}<0.0001$ respectively.

Conclusion: AECOPD associated with pleuritic chest pain, immobility, high DD, should be considered for PE. Chest-ultrasound, as a low-cost and safe procedure, can be a very helpful investigation.

Key words: COPD, D-dimer, pulmonary embolism, deep vein thrombosis, chest ultrasonography, lung computed tomography angiography
\end{abstract}

Med Res J 2020; 5 (2): 79-85

\section{Introduction}

COPD is a major health burden worldwide. It is the fourth-leading cause of mortality, with more than 3 million deaths annually and by 2020, COPD from fourth will become the third-world leading cause of death. COPD is a systemic disease with many comorbidities like cardiovascular disease, anemia, polycythemia, malnutrition, muscle disorder, osteoporosis, metabolic syndrome, diabetes, gastroesophageal reflux, anxiety, depression, hormonal imbalance, infections, lung cancer, thrombosis [1]. Exacerbations of COPD are the episodic periods of the disease, characterized by deterioration of respiratory function, requiring treatment with steroids and/or antibiotics. Most deaths caused by COPD appear to occur during exacerbations. AECOPD is a frequent 
reason for a visit to the emergency department and hospitalization in medical wards. Respiratory infections are responsible for $50-70 \%$ of COPD exacerbations and environmental pollution causes another $10 \%$. Nearly $30 \%$ of all COPD exacerbations have unknown etiology $[1,2]$. Many studies have shown that COPD is a moderate and independent factor for PE. Patients with COPD are at high risk for $P E$ because of systemic inflammation, limited mobility and co-existing comorbidities. PE can exacerbate respiratory symptoms such as dyspnea and chest pain [3]. The frequency of PE in patients with acute exacerbation of COPD remains uncertain [4]. Studies suggest that up to $30 \%$ of patients seen as emergencies with exacerbations of COPD may have DVT or PE $[5,6]$. The prevalence of PE in post-mortem studies ranges from $28 \%$ to $51 \%$ [2]. Patients with COPD have almost double the risk of PE and other venous thromboembolic incidents than those with no COPD [7]. It was proved that DD is still the most useful test to exclude VTE with a negative predictive value of $98 \%$. An elevated DD gives a more precise risk assessment for VTE when combined with a clinical scoring system like the revised Geneva score [8]. DD measurement is a simple and rather noninvasive test that rules out $\mathrm{PE}$ without the need for additional imaging procedures; unfortunately, there is still a debate about the efficacy of DD tests in diagnosing PE in patients suffering from acute exacerbation of COPD [9]. Irrespective of the presence of venous thromboembolism, DD levels are increased in patients with COPD exacerbation. With the advent of CTPA, it is now possible to visualise the clot by an imaging technique and reliably confirm the diagnosis of PE in COPD subjects. This technique carries a high risk in some patients who are allergic to the contrast agent or have renal impairment with rtheisk of contrast nephropathy. Additionally, radiation exposure, high cost of CTPA, necessitates the search for a tool to reduce the need for unnecessary radiological investigation [10]. TUS in detecting PE with high sensitivity and diagnostic accuracy, is a non-invasive, widely available, cost-effective method which can be rapidly performed. A negative TUS study cannot rule out PE with certainty, but positive TUS findings with moderate/high suspicion for PE may prove a valuable tool in the diagnosis of $\mathrm{PE}$, especially at emergency setting, for critically ill and immobile patients, facilitating immediate treatment decision [11]. TUS could suggest the diagnosis of PE by the presence of one or more typical pleural-based hypoechoic lesions that could be associated with pleural effusion or not [12]. Increased expression of VTE as PE in COPD patients may be problematic since the mortality of COPD patients with PE is particularly high, and COPD has been integrated with prognostic scores such as the Simplified Pulmonary Embolism Severity Index [13]. COPD has also been associated with inap- propriate management in the case of suspected PE and the suggestion of PE may be challenging in COPD patients because of the similarities in symptoms [14].

\section{Objective}

The objective of the present study was to evaluate the prevalence and risk factors for PE and DVT in hospitalized patients with AECOPD.

\section{Material and methods}

Design and setting: Single-center, prospective, observational trial of 100 hospitalized patients with AECOPD (72 men and 28 women, aged $40-75$ years, mean age $62.2 \pm 6.8$ ) who were hospitalized in the twoyear period 2018-2020 at Department of Pulmonology and Allergology at General Hospital "8mi Septemvri" in Skopje. AECOPD was diagnosed according to the recommendations of Global Initiative for Chronic Obstructive Lung Disease (GOLD). Our local ethics committee approved the study protocol. All study subjects were informed about the study and their written consent was obtained.

Investigations: Prospectively included patients with AECOPD were investigated by a diagnostic algorithm for PE. In first 48-hours of hospital admission was performed - collecting data for full medical histories; body weight with calculated body mass index (BMI), smoking history, clinical prediction rules for PE - revised Geneva score and Wells criteria [15, 16], ECG, a blood sample for laboratory and arterial blood gas analysis, spirometry, serum DD, chest-X-ray, DULE, TUS, echocardiography. Patients with high DD and DVT or high DD and abnormal TUS underwent CTPA.

Inclusion criteria: COPD patients hospitalized because of AECOPD (diagnosed according to the recommendations of GOLD, as acute deterioration from a stable respiratory condition, which required hospitalization), age 40-75, both genders, current or former smokers, normal liver and kidney function (according to laboratory results - aspartate aminotransferase 2-35 U/L, alanine aminotransferase 2-45 U/L, total bilirubin $<1.2 \mathrm{mg} / \mathrm{dL}$, gamma-glutamyl transferase 9-48 U/L, alkaline phosphatase 44-147 U/L blood urea nitrogen $<8.3 \mathrm{mmol} / \mathrm{L}$, serum creatinine 53-115 $\mu \mathrm{mol} / \mathrm{L}$ and previous patient medical history), non-allergic to contrast, signed informed consent.

Exclusion criteria: asthma, pneumothorax, lung fibrosis, active tuberculosis, sarcoidosis, malignancies, hepatic, renal diseases, coagulation disorders, haematological diseases, congenital heart disease, myocardial infarction during the previous year (while antiplatelet therapy is necessary), regular therapy with 
oral anticoagulant or antiplatelet medication, collagen vascular diseases, surgery in the previous 3 months, transfusion of blood or blood component in the previous 3 months, not willing to participate.

AECOPD subjects were stratified based on medical history before exacerbation and spirometry (stages I-IV) according to GOLD criteria. Then, the patients were divided into 2 sub-groups: patients with $A E$ COPD of known etiology and patients with AECOPD of undetermined etiology. AECOPD of known etiology tracheobronchitis, pneumonia discontinuation of COPD inhaler therapy or oxygen treatment, refused scheduled pulmonary rehabilitation, problems in nutritional status and congestive heart failure.

Patients were also classified according to the result of CTPA in PE-diagnosed subgroup and non-PE AECOPD patients.

In the first 48-hours after hospital admission patients were classified using Wells and revised Geneva criteria as low, moderate and high risk for pulmonary emboli. The score comprised 8 variables (points): age older than 65 years (1 point), previous deep venous thrombosis or pulmonary embolism (3 points), surgery or fracture within 1 month (2 points), active malignant condition (2 points), unilateral lower limb pain (3 points), hemoptysis (2 points), heart rate of 75 to 94 beats $/ \mathrm{min}$ (3 points) or 95 beats/min or more (5 points), and pain on lower-limb deep venous palpation and unilateral edema (4 points). Low-probability category (0 to 3 points), intermediate-probability category (4 to 10 points), and high-probability category ( $>$ or $=11$ points) $[15,16]$.

Laboratory analysis included routine biochemistry tests for liver, kidney function, glucose level, acutephase reactants (white blood cell count, erythrocyte sedimentation rate, C-reactive protein), troponin and DD.

DD was measured with DD-assay system (Boehringer, Germany), which is a particle-enhanced immunoturbidimetric assay, level below $0.5 \mathrm{mcg} / \mathrm{mL}$ was considered to be within the normal range.

Arterial blood gas analyses were performed with SIEMENS RAPIDPOINT 405 System (Siemens Healthineers, Australia). Interpretation of the results: hypercapnia $\mathrm{PaCO} 2>45 \mathrm{mmHg}$, hypocapnia $\mathrm{PaCO} 2<35 \mathrm{mmHg}$, acidosis $\mathrm{pH}<7.35$, alkalosis $\mathrm{pH}>7.45$, hypoxemia $\mathrm{PaO} 2<80 \mathrm{mmHg}$ (mild $60-80 \mathrm{mmHg}$, moderate 40-59 $\mathrm{mmHg}$, severe $<40 \mathrm{mmHg}$ ).

Pre-bronchodilator (baseline) spirometry measured forced vital capacity (FVC), forced expiratory volume in one second (FEV1), FEV1/FVC ratio, maximal expiratory flow at $75 \%, 50 \%, 25 \%$, and $25-75 \%$ of FVC by electronic spirometer Spirobank G USB Spirometer (Medical International Research, Roma, Italy). The results of measurements were expressed as percentages of the predicted values following the actual recommendations of the European Respiratory Society (ERS) and the American Thoracic Society (ATS). Post-bronchodilator spirometry was performed 20 minutes after administration of $400 \mathrm{mcg}$ salbutamol and fixed airflow narrowing was considered if post-bronchodilator FEV1/FVC value remained less than 0.70 .

DULE was performed using General Electric Vivid 7 ultrasound unit, linear transducer $(6-10 \mathrm{MH})$, starting from a common femoral vein and including the calf vein. Lack of compressibility was considered to indicate deep venous thrombosis.

TUS was performed by a chest physician with General Electric Vivid 7 ultrasound device using a convex probe $(3.5 \mathrm{MHz})$. If the patient had chest pain, the examination started from the painful area and then all intercostal areas were obtained in six vertical lines (paravertebral, midscapular, posterior axillary, midaxillary, anterior, axillary, and midclavicular). Findings were described like pleural-based hypoechoic lesions (wedge-shaped, round or triangular) that could be associated with pleural effusion or not, nonspecific subpleural hypoechogenic lesion and normal finding. Presence of pleural effusion without subpleural changes or normal ultrasonography finding were accepted as negative TUS for PE.

CTPA was performed to show emboli in the lung with a 16-section multidetector CT scanner (GE Light Speed 16; GE Healthcare, Milwaukee, Wisconsin, USA) within $24 \mathrm{~h}$ of admission). Patients with high DD and DVT or high DD and abnormal TUS underwent CTPA. CT-scans for thorax were obtained in the craniocaudal direction during a single inspiratory breath hold from the apex to the diaphragm after injection of $2 \mathrm{~mL} / \mathrm{kg}$ (maximum $150 \mathrm{~mL}$ ) of nonionic contrast lopromide (Ultravist-300, Bayer Pharma), through an arm vein. Reconstruction interval was $2 \mathrm{~mm}$. PE was diagnosed if contrast material outlined an intraluminal defect present in at least two consecutive images, or if the vessel was occluded by low attenuation material. The localization of the thrombi was noted.

Cardiac function was evaluated with ECG and resting 2D Doppler Echocardiography (performed by a cardiologist using General Electric Vivid 7, according to the recommendations of the American Heart Association), to detect direct and indirect signs of pulmonary emboli. Echocardiography finding of right ventricular dilation (when Right Ventricular End Diastolic dimension - RVEDd exceeded the normal range of $0.9-2.6 \mathrm{~cm}$ ), leftward moving of interventriculer septum, pulmonary hypertension (defined as a systolic Pulmonary Artery Pressure - sPAP value equal to or higher than $30 \mathrm{mmHg}$ ), hypokinesis of the right ventricle. Sinus tachycardia, atrial fibrillation, $\mathrm{T}$ wave abnormalities, pattern, bundle branch block, right axis deviation, S1Q3T3 on ECG. 


\section{Statistical analysis}

Statistical analysis was done using the Statistical Package for the Social Sciences (SPSS) version 17.0 for Windows (SPSS. Inc., Chicago, IL, USA). Continuous variables were expressed as mean values with standard deviation (SD). Most of the results were with numerical values and Pearson Correlation test was performed for comparison. The nominal variables were presented as numbers and percentages. The level of P-value less than 0.05 was considered as statistically significant.

\section{Results}

A total of 100 hospitalized patients with AECOPD, according to the inclusion and exclusion criteria were enrolled as the study group. The investigated group included 62 men, 38 women, age 40-75. PE was diagnosed in $26(26.0 \%)$ of investigated group and DVT in $5(5.0 \%)$. According to the presence of pulmonary embolism on CTPA, patients were divided in two groups: with PE $(n=26)$ and non-PE patients $(n=74)$. There was no statistically significant difference between patients with PE and without, according to age, gender, spirometry and comorbidities $(P>0.05)$. Immobility and obesity were significantly higher among patients with $P E, P=0.032$ and $P<0.0001$ respectively. Characteristics of the study subjects are presented in Table
1. According to the revised Geneva score, 55 (55.0\%) of all 100 patients had a low probability of PE and in $10(18.2 \%)$ of these 55 patients, PE was confirmed. Forty-two patients (42.0\%) of the investigated group, had an intermediate probability of PE and 25 (59.5\%) of them, had confirmed PE. Three of 100 investigated patients had a high probability of PE and in all of them (100.0\%), PE was diagnosed. Previous DVT or PE were significantly more prevalent in PE group than non-PE group $(23.1 \%$ vs. $10.8 \%, \mathrm{P}<0.05)$ (Tab. 2$)$.

Frequency of PE proportionally increased in $\mathrm{AE}$ COPD patients as FEV1 declined, presented in Table 3.

According to AECOPD etiology, patients were divided into 2 sub-groups: patients with AECOPD of known etiology and patients with AECOPD of undetermined etiology presented in Table 4.

Patients with pleuritic chest-pain, abnormal TUS, DVT and high DD were more likely to develop PE. DD was significantly higher among patients with $P E$ than those without $(3.34 \pm 1.1 \mu \mathrm{g} / \mathrm{mL}$ vs. $2.2 \pm 0.8 \mu \mathrm{g} / \mathrm{mL}$, $\mathrm{P}<0.0001)$. There was correlation between the presence of $P E$ and elevated DD $>2.0 \mu \mathrm{g} / \mathrm{mL}(P=0.02)$. The presence of DVT in investigated group of 100 patients was $5(5.0 \%)$ and all of them were diagnosed with PE (PE group, $n=26$ ). DVT was significantly higher in $P E$ group vs. non-PE group $(P<0.005)$. Electrocardiographic abnormalities such as atrial fibrillation or right bundle branch block appeared more often, but the S1Q3T3 pattern appeared less often. They also

Table 1. Characteristics of the study subjects

\begin{tabular}{|c|c|c|c|}
\hline Characteristic & $\begin{array}{l}\text { Group } 1 \\
\text { (PE group) } \\
(\mathrm{n}=26)\end{array}$ & $\begin{array}{l}\text { Group } 2 \\
\text { (non-PE group) } \\
(n=74)\end{array}$ & $P$ value \\
\hline $\begin{array}{l}\text { Gender } \\
\text { Male } \\
\text { Female }\end{array}$ & $\begin{array}{l}17(65.4 \%) \\
9(34.6 \%)\end{array}$ & $\begin{array}{l}45(60.8 \%) \\
29(39.2 \%)\end{array}$ & $P=0.6792$ \\
\hline $\begin{array}{l}\text { Mean age } \\
\text { Male } \\
\text { Female }\end{array}$ & $\begin{array}{l}62.9 \pm 6.5 \\
63.9 \pm 6.1\end{array}$ & $\begin{array}{l}61.3 \pm 8.1 \\
64.3 \pm 7.4\end{array}$ & $\begin{array}{l}P=0.3657 \\
P=0.8051\end{array}$ \\
\hline $\begin{array}{l}\text { Smoking status } \\
\text { Current smokers } \\
\text { Ex-smokers } \\
\text { Pack-year smoked }\end{array}$ & $\begin{array}{l}16(61.5 \%) \\
10(38.5 \%) \\
56.3 \pm 20.8\end{array}$ & $\begin{array}{l}50(67.6 \%) \\
24(32.4 \%) \\
54.7 \pm 19.5\end{array}$ & $P=0.5741$ \\
\hline Mean BMI value & $30.8 \pm 4.9$ & $25.9 \pm 3.1$ & $P<0.0001$ \\
\hline $\begin{array}{l}\text { Mean baseline spirc } \\
\text { FVC }(\% \text { pred }) \\
\text { FEV }_{1}((\% \text { pred }) \\
\text { FEV }_{1} / \text { FVC ratio }\end{array}$ & $\begin{array}{l}66.8 \pm 10.1 \\
45.5 \pm 15.9 \\
0.58 \pm 0.05\end{array}$ & $\begin{array}{l}67.2 \pm 9.7 \\
47.4 \pm 15.4 \\
0.60 \pm 0.05\end{array}$ & $\begin{array}{l}P=0.8583 \\
P=0.5927 \\
P=0.0825\end{array}$ \\
\hline $\begin{array}{l}\text { Blood gas analysis } \\
\mathrm{PaO} 2(\mathrm{kPa}) \\
\mathrm{PaCO} 2(\mathrm{kPa}) \\
\text { O2 saturation }(\%)\end{array}$ & $\begin{array}{l}8.1 \pm 1.1 \\
5.5 \pm 1.8 \\
84.6 \pm 5.3\end{array}$ & $\begin{array}{l}8.3 \pm 1.6 \\
5.1 \pm 1.7 \\
86.6 \pm 7.3\end{array}$ & $\begin{array}{l}P=0.5570 \\
P=0.2975 \\
P=0.2030\end{array}$ \\
\hline
\end{tabular}

BMI — body mass index; FVC — forced vital capacity; FEV1 — forced expiratory volume in one second; \% pred — percentage of the predicted value; $\mathrm{PaO} 2$ - Partial Pressure of Oxygen; $\mathrm{PaCO} 2$ - Partial Pressure of Carbon dioxide; O2 - Oxygen; $\mathrm{kPa}-\mathrm{Kilopascal}$ 
Table 2. Study sample characteristics according to The Revised Geneva Score [16]

\begin{tabular}{|c|c|c|c|}
\hline Variable & $\begin{array}{c}\text { Group } 1 \text { (PE group) } \\
(\mathrm{n}=26)\end{array}$ & $\begin{array}{c}\text { Group } 2 \text { (non-PE group) } \\
(\mathrm{n}=74)\end{array}$ & $P$ value \\
\hline \multicolumn{4}{|l|}{ Risk factors } \\
\hline Age $>65$ years & $14(53.8 \%)$ & 35 (47.3\%) & $P=0.5704$ \\
\hline Previous DVT or PE & $5(19.2 \%)$ & $4(5.4 \%)$ & $P=0.0352$ \\
\hline Recent surgery & $\begin{array}{l}\text { Not applicable } \\
\text { (exclusion criteria) }\end{array}$ & $\begin{array}{c}\text { Not applicable } \\
\text { (exclusion criteria) }\end{array}$ & 1 \\
\hline Active malignant condition & $\begin{array}{l}\text { Not applicable } \\
\text { (exclusion criteria) }\end{array}$ & $\begin{array}{l}\text { Not applicable } \\
\text { (exclusion criteria) }\end{array}$ & 1 \\
\hline \multicolumn{4}{|l|}{ Symptoms } \\
\hline Unilateral lower-limb pain & $5(19.2 \%)$ & $3(2.7 \%)$ & $P=0.0047$ \\
\hline Hemoptysis & 7 (26.9\%) & $0(0.0 \%)$ & $P<0.0001$ \\
\hline \multicolumn{4}{|l|}{ Clinical signs } \\
\hline $\begin{array}{l}\text { Heart rate: } 75-94 \text { beats } / \mathrm{min} \\
\geq 95 \text { beats } / \mathrm{min}\end{array}$ & $23(88.5 \%)$ & 45 (60.8\%) & $P=0.0096$ \\
\hline $\begin{array}{l}\text { Pain on lower-limb venous } \\
\text { palpation, unilateral edema }\end{array}$ & $4(15.4 \%)$ & $2(2.6 \%)$ & $P=0.0180$ \\
\hline \multicolumn{4}{|l|}{ Clinical probability } \\
\hline Low risk ( 0 to 3 points, $n=55$ ) & $10(18.2 \%)$ & $45(81.8 \%)$ & $P<0.0001$ \\
\hline $\begin{array}{l}\text { Intermediate risk (4 to } 10 \text { points, } \\
n=42 \text { ) }\end{array}$ & 25 (59.5\%) & 17 (40.5\%) & $P=0.0958$ \\
\hline High risk ( $>$ or $=10$ points, $n=3$ ) & $3(100.0 \%)$ & $0(0.0 \%)$ & $P<0.0001$ \\
\hline
\end{tabular}

DVT — deep vein thrombosis; PE — pulmonary embolism

Table 3. Distribution AECOPD patients with diagnosed Pulmonary Embolism (PE) based on the degree of airflow limitation

\begin{tabular}{lc}
\hline COPD severity & $\begin{array}{c}\text { Patients with } \\
\text { diagnosed PE (n = 26) }\end{array}$ \\
\hline GOLD 1 (FEV1 $\geq 80 \%$ pred.) & $0(0.0 \%)$ \\
GOLD 2 (FEV1 = 50-79\% pred.) & $3(11.5 \%)$ \\
GOLD 3 (FEV1 = 30-49\% pred.) & $8(30.7 \%)$ \\
GOLD 4 (FEV1 < 30\% pred.) & $15(57.7 \%)$ \\
\hline
\end{tabular}

COPD - chronic obstructive pulmonary disease; PE — pulmonary embolism; GOLD - Global Initiative for Chronic Obstructive Lung Disease; FEV1 - forced expiratory volume in one second; \% pred percentage of the predicted value

had a higher frequency of hypoxemia and hypercapnia. Hospital stay duration was longer in PE group (14 \pm 4 days in PE group vs. $9 \pm 2$ days in non-PE group, $P<0.0001)$. The presence of symptoms of respiratory infection was less common in PE group, in $13(50.0 \%)$ vs. 65 (87.8\%) of non-PE group ( $P=0.0001$, $95 \% \mathrm{Cl} 17.5603$ to $56.6053 \%$ ). TUS was true positive in $24(24.0 \%)$ patients, true negative in 54 (54.0\%), false positive in $20(20.0 \%)$ and false-negative in 2 (2.0\%). The results of the localization of pulmonary emboli on CTPA and TUS findings are presented in Table 5.
Table 4. Etiology of acute COPD exacerbation AECOPD etiology of hospitalized patients $(n=100)$ Known etiology 68 (68.0\%)

Tracheobronchitis $25(25.0 \%)$

Pneumonia

$20(20.0 \%)$

Discontinuation of COPD inhaler therapy $15(15.0 \%)$

Congestive heart failure $\quad 8(8.0 \%)$

Undetermined etiology 32 (32.0\%)

COPD - chronic obstructive pulmonary disease; AECOPD - Acute Exacerbation of Chronic Obstructive Pulmonary Disease

\section{Discussion}

Clinical diagnosis of acute PE is difficult in patients with COPD. Pulmonary embolism resembles COPD exacerbation so closely that these two entities are often impossible to distinguish clinically. The reported incidence of PE in studies done postmortem of patients with COPD ranges from $28 \%$ to $51 \%$ [17]. But the prevalence of $\mathrm{PE}$ in studies performed in living patients with COPD is lower than when autopsy is done, ranging from $3-25 \%$. Since PE can lead to cough and dyspnea, similarly to infectious events, there is a difficulty 
Table 5. Results of Computed Tomography with Pulmonary Angiography (CTPA) and Thoracic Ultrasonography (TUS) findings

\begin{tabular}{|c|c|c|c|}
\hline \multicolumn{4}{|c|}{ Analysis of CTPA and TUS in PE group $(n=26)$} \\
\hline \multicolumn{2}{|c|}{ Localization of PE on CTPA } & \multicolumn{2}{|c|}{ TUS findings in PE group } \\
\hline Main pulmonary artery & $7(26.9 \%)$ & $\begin{array}{l}\text { Pleural-based hypoech } \\
\text { lesions with/or without } \\
\text { pleural effusion }\end{array}$ & $\begin{array}{l}\text { Total } 15(57.7 \%) \\
\text { with pleural effusion } 8(30.8 \%) \\
\text { without pleural effusion } 7(26.9 \%)\end{array}$ \\
\hline Lobar and interlobar arteries & $10(38.5 \%)$ & $\begin{array}{l}\text { Nonspecific subpleural } \\
\text { hypoechogenic lesion }\end{array}$ & $9(34.6 \%)$ \\
\hline Subsegmental arteries & $9(34.6 \%)$ & Normal finding & $2(7.7 \%)$ \\
\hline
\end{tabular}

CTPA — computed tomography with pulmonary angiography; TUS — thoracic ultrasonography; PE — pulmonary embolism

in excluding lower respiratory tract infection. Nearly $30 \%$ of all exacerbations of COPD do not have a clear etiology [18]. The most common causes of exacerbation are infection of the tracheobronchial tree and air pollution, but in up to $30 \%$ of exacerbations, the specific cause cannot be identified. Although PE can exacerbate respiratory symptoms such as dyspnea and chest pain, and COPD patients are at high risk for $P E$ due to a variety of factors including limited mobility, inflammation, and comorbidities, the prevalence of PE during exacerbations is uncertain. Generally, the prevalence of VTE including PE has been found to be markedly lower in Asians than in whites and African Americans [18, 19]. According to RIETE registry, COPD patients presenting with $P E$ have an increased risk for PE recurrences and fatal PE compared with those presenting with DVT alone [20].

Our study aimed to assess the prevalence and risk factors for PE and DVT in hospitalized patients with AECOPD. We performed a single-center, observational, prospective study, including 100 patients with AECOPD (62 male, 38 female), aged 40 to 75 years, diagnosed according to the actual GOLD criteria, stratified according to airflow limitation (I-IV), divided into subgroups (PE-diagnosed and non-PE, with known and undetermined exacerbation etiology). PE was diagnosed in $26(26.0 \%)$ and DVT in $5(5.0 \%)$ of the investigated group. In August 2019, Hassen et al. presented the results of prospective cohort study about the incidence of $P E$ in severe AECOPD requiring mechanical ventilation. The incidence of PE was $13.7 \%$ and was associated with high mortality [3]. The same result of PE prevalence $13.7 \%$, reported Gunen et al. in 2010 [10]. Choi et al. [18] in 2013, published a study of the prevalenceof PE and DVT in Koreans with COPD exacerbation, and the result was $5.0 \%$, confirming the lower prevalence of $\mathrm{PE}$ in the Asian population. These results are lower than our study, but Akpinar et al. [2] in 2014 published the results of a study performed in Ankara, Turkey with the prevalence of PE in AECOPD 29.1\%. Tillie-Leblond et al. [17] also found the prevalence of PE to be $25.0 \%$ in COPD patients with severe AECOPD of unknown etiology. Pang et al. [21] in 2018 reported DVT in AECOPD in $5.6 \%$ of the patients, similar to our study $5.0 \%$. In our study, patients with pleuritic chest pain, abnormal chest-ultrasound, phlebitis on DULE and high DD were more likely to develop PE. DD was significantly higher among patients with $\mathrm{PE}$ than those without (3.34 $\pm 1.1 \mu \mathrm{g} / \mathrm{mL}$ vs. $2.2 \pm 0.8 \mu \mathrm{g} / \mathrm{mL}, \mathrm{P}<0.0001)$. This finding is in agreement with Akpinar et al. [2] who found significantly higher DD levels in COPD patients than control subjects, and the hospital stay duration was longer in the patients with PE than in those without, like in our study. Choi et al. [18] in contrast presented no difference in hospital stay duration. Our results about localization of pulmonary emboli on CTPA, showed higher prevalence of emboli in main, lobar and interlobar arteries than peripheral (subsegmental) localization. This finding is similar to the results of Choi et al. [18] but in contrast with Akpinar et al. [2], where peripheral localization was more frequent.

The results of the gas analysis showed no significant difference between the group with PE and without, similar to Gunen et al. [11]. Akpinar et al. [2] presented respiratory alkalosis and hypocapnia were more common in patients with COPD exacerbation accompanying PE.

TUS was applied to all patients in this study and high probability for $\mathrm{PE}$ was the presence of at least one typical subpleural hypoechoic lesion with or without pleural effusion. TUS was true positive in 24 (24.0\%) and false-negative in $2(2.0 \%)$ which is similar to the results published by Baz et al. [22] in 2019 that TUS with its high specificity and diagnostic accuracy should be widely performed in AECOPD patients.

\section{Conclusions}

This trial shows that $P E$ is not uncommon in our population and one of four COPD patients who require hospitalization for AECOPD may have PE, especially cases with unexplained respiratory exacerbation, immobility, high DD. Clinical manifestations of PE like dyspnoea, pleuritic chest pain are nonspecific, and easily could 
be underestimated in subjects with COPD, which leads to disease worsening, delay of anticoagulant therapy and higher mortality rate. Thoracic ultrasonography as a low-cost and safe procedure can be a very helpful investigation.

The findings of the present study are subjects of some limitations. First, only one center is included and relatively small size of the study subjects could have certain implications on evaluated data and its interpretation. Second, the unequal distribution of COPD patients by degree of airflow limitation could have certain influence on data obtained and its interpretation. On the other side, the strength of the study is the diagnostic assessment of venous thromboembolism (PE and DVT) in hospitalized AECOPD patients, using clinical risk assessment, TUS, CTPA and DULE.

\section{Conflict of interest}

The authors report no competing interests.

\section{References}

1. Global Initiative for Chronic Obstructive Lung Disease (GOLD). Executive Summary: Global Strategy for Diagnosis, Management, and Prevention of COPD - Updated 2020.

2. Akpinar EE, Hoşgün D, Akpinar $S$, et al. Incidence of pulmonary embolism during COPD exacerbation. J Bras Pneumol. 2014; 40(1): 38-45, doi: 10.1590/S1806-37132014000100006, indexed in Pubmed: 24626268

3. Hassen MF, Tilouche N, Jaoued O, et al. Incidence and Impact of Pulmonary Embolism During Severe COPD Exacerbation. Respir Care 2019: 64(12): 1531-1536, doi: 10.4187/respcare.06661, indexed in Pubmed: 31387893.

4. Couturaud F, Bertoletti L, Sanchez O, et al. Frequency Pulmonary embolism (PE) in patients with an acute Exacerbation of chronic obstructive Pulmonary disease (COPD). PEP prospective trial. Eur Respir J. 2019; 54(63): PA3637.

5. Perrier A, Perneger T, Cornuz J, et al. COPD-PE study. [The COPD-PE study: prevalence and prediction of pulmonary embolism in acute exacerbations of chronic obstructive pulmonary disease]. Rev Mal Respir. 2004; 21(4 Pt 1): 791-796, doi: 10.1016/s0761-8425(04)714205, indexed in Pubmed: 15536380.

6. Tompkins R, Harris V, Brown C, et al. Diagnosing Pulmonary Embolism in Patients with Suspected or Established Chronic Lung Disease. The Southwest Respiratory and Critical Care Chronicles. 2014; 2(8): 5, doi: 10.12746/swrccc.v2i8.172.

7. MacNee W. Systemic inflammatory biomarkers and co-morbidities of chronic obstructive pulmonary disease. Ann Med. 2013; 45(3): 291-300 doi: 10.3109/07853890.2012.732703, indexed in Pubmed: 23110517.
8. Szturmowicz M, Kacprzak A, Wyrostkiewicz D, et al. Non-high risk $P E$ in the patients with acute or exacerbated respiratory disease: the value of the algorithm based on D-dimer evaluation and Revised Geneva Score. Pneumonol Alergol Pol. 2015; 83(6): 445-452, doi: 10.5603/PiAP.2015.0073, indexed in Pubmed: 26559797.

9. Silva DR, Coelho AC, Gazzana MB, et al. D-dimer levels in stable COPD patients: a case-control study. COPD. 2012; 9(4): 426-431, doi: 10.3109/15412555.2012.683840, indexed in Pubmed: 22612665.

10. Gunen H, Gulbas G, In E, et al. Venous thromboemboli and exacerbations of COPD. Eur Respir J. 2010; 35(6): 1243-1248, doi: 10.1183/09031936.00120909, indexed in Pubmed: 19926740.

11. Comert SS, Caglayan B, Akturk U, et al. The role of thoracic ultrasonography in the diagnosis of pulmonary embolism. Ann Thorac Med. 2013; 8(2): 99-104, doi: 10.4103/1817-1737.109822, indexed in Pubmed: 23741272

12. Reissig A, Copetti R, Kroegel C. Current role of emergency ultrasound of the chest. Crit Care Med. 2011; 39(4): 839-845, doi: 10.1097/CCM.0b013e318206d6b8, indexed in Pubmed: 21263325.

13. Jiménez D, Aujesky D, Moores L, et al. RIETE Investigators. Simplification of the pulmonary embolism severity index for prognostication in patients with acute symptomatic pulmonary embolism. Arch Intern Med 2010; 170(15): 1383-1389, doi: 10.1001/archinternmed.2010.199, indexed in Pubmed: 20696966.

14. Bertoletti L, Quenet S, Mismetti $P$, et al. RIETE Investigators. Clinical presentation and outcome of venous thromboembolism in COPD. Eur Respir J. 2012; 39(4): 862-868, doi: 10.1183/09031936.00058811, indexed in Pubmed: 21885395.

15. Le Gal G, Righini M, Roy PM, et al. Prediction of pulmonary embolism in the emergency department: the revised Geneva score. Ann Intern Med. 2006; 144(3): 165-171, doi: 10.7326/0003-4819-144-3-20060207000004, indexed in Pubmed: 16461960.

16. Wells PS, Anderson DR, Rodger M, et al. Excluding pulmonary embolism at the bedside without diagnostic imaging: management of patients with suspected pulmonary embolism presenting to the emergency department by using a simple clinical model and d-dimer. Ann Intern Med. 2001; 135(2): 98-107, doi: 10.7326/0003-4819-1352-200107170-00010, indexed in Pubmed: 11453709.

17. Tillie-Leblond I, Marquette $\mathrm{CH}$, Perez T, et al. Pulmonary embolism in patients with unexplained exacerbation of chronic obstructive pulmonary disease: prevalence and risk factors. Ann Intern Med. 2006; 144(6): 390-396, doi: 10.7326/0003-4819-144-6-200603210-00005, indexed in Pubmed: 16549851.

18. Choi KJ, Cha SI, Shin KM, et al. Prevalence and predictors of pulmonary embolism in Korean patients with exacerbation of chronic obstructive pulmonary disease. Respiration. 2013; 85(3): 203-209, doi: 10.1159/000335904, indexed in Pubmed: 22338649

19. Rizkallah J, Man SF, Sin DD. Prevalence of pulmonary embolism in acute exacerbations of COPD: a systematic review and metaanaIysis. Chest. 2009; 135(3): 786-793, doi: 10.1378/chest.08-1516, indexed in Pubmed: 18812453.

20. Bertoletti L, Quenet S, Laporte S, et al. RIETE Investigators. Pulmonary embolism and 3-month outcomes in 4036 patients with venous thromboembolism and chronic obstructive pulmonary disease: data from the RIETE registry. Respir Res. 2013; 14: 75, doi: 10.1186/14659921-14-75, indexed in Pubmed: 23865769.

21. Pang H, Wang L, Liu J, et al. The prevalence and risk factors of venous thromboembolism in hospitalized patients with acute exacerbation of chronic obstructive pulmonary disease. Clin Respir J. 2018; 12(11): 2573-2580, doi: 10.1111/crj.12959, indexed in Pubmed: 30207643.

22. Baz A, Hamdy I, Mohammed A, et al. Diagnostic validity of thoracic ultrasound in the assessment of pulmonary embolism. Egypt J Radiol Nucl Med. 2019; 50(1), doi: 10.1186/s43055-019-0005-z. 